\section{Formación integral y profesionalismo médico: una propuesta de trabajo en el aula}

\author{
J.C. Morales-Ruiz
}

\section{Introducción}

La formación integral es uno de los pilares fundamentales de la preparación de los estudiantes universitarios para un ejercicio ciudadano y profesional exitoso. Esta consideración adquiere especial relevancia cuando se trata de la formación de los médicos, profesionales responsables del cuidado de la salud y de la vida de los seres humanos, hecho que implica una mayor responsabilidad frente a la comunidad médica y a la sociedad en general.

Desde una perspectiva un tanto simplista, aunque no por ello carente de profundidad, el objetivo fundamental de la educación médica está orientado a la formación de profesionales preparados para servir a 'los propósitos fundamentales de la medicina, incluyendo el mantenimiento de la salud, el alivio del sufrimiento, la curación de la enfermedad, el control de la pérdida innecesaria de vidas y el acompañamiento durante el tránsito hacia una muerte digna, acciones que se encuentran enmarcadas en las cláusulas del 'contrato social' que se establece, en forma tácita o expresa, entre el médico que ejerce su profesión y la comunidad que demanda sus servicios [1].

En el mismo sentido, Wojtczak afirma que '... la medicina es un cometido moral, una profesión cuyos miembros comulgan con una serie de principios válidos desde el inicio de sus actividades, como el respeto hacia los demás, la empatía, la compasión, la honestidad, la integridad, el altruismo y la excelencia profesional', y hace énfasis en el hecho de que estos principios constituyen las 'raíces del profesionalismo médico y las bases del contrato colectivo con la sociedad, que tradicionalmente ha hecho de la medicina algo diferente de otras profesiones u ocupaciones' [2].

Desde esa perspectiva, y teniendo en cuenta la magnitud del compromiso social que implica el ejercicio de la medicina y el proceso de formación de los futuros médicos, el trabajo llevado a cabo en las instituciones universitarias no puede limitarse a la transmisión de conocimientos científicos ni al desarrollo de habilidades técnicas, sino que ha de garantizar el desarrollo armónico de todas las dimensiones del individuo, involucrando en el proceso los elementos científicos, tecnológicos, éticos, culturales, sociales y humanísticos requeridos para la consolidación progresiva del profesionalismo médico.

A la luz de los conceptos expresados, el proceso de formación integral se halla ligado de forma indisoluble al desarrollo del profesionalismo médico. Por tal razón, las facultades de medicina tienen la obligación de concienciar a sus estudiantes acerca de los principios y valores que regulan la práctica médica desde los primeros tiempos y de proporcionarles las herramientas necesarias para reconocer y afrontar en forma adecuada los conflictos existentes entre las más elevadas tradiciones de la profesión y los imperativos de sistemas sanitarios con una clara orientación por las normas del mercado.

En consonancia con lo anterior, el doctor Jordan Cohen, ex presidente de la Asociación de Colegios Médicos Americanos, en una de sus alocuciones a la convención anual, afirma que 'es en estos momentos una obligación de la profesión médica el cultivar los valores nucleares del profesionalismo en los futuros médicos... y
Decano de la

Facultad de Medicina. Universidad de Ciencias Aplicadas y Ambientales. Bogotá, Colombia.

Correspondencia Dr. Juan Carlos Morales Ruiz. Facultad de Medicina. Universidad de Ciencias Aplicadas y Ambientales. Calle 222, n. ${ }^{\circ}$ 55-37. Bogotá DC, Colombia.

E-mail

juancarlosmoralesruiz@ gmail.com 
mantenerse firme en el soporte de estos valores que hacen de nuestra profesión una profesión honorada y honorable' [2].

\section{La formación integral}

A pesar de que existe una tendencia generalizada a considerar la formación integral como referente de los proyectos educativos de las instituciones de educación superior, es posible encontrar diferentes interpretaciones, especialmente en la materialización de dicho concepto en la práctica cotidiana, hecho que genera dificultades para la implementación de estrategias orientadas al cumplimiento de tal propósito y para la evaluación objetiva de los resultados obtenidos al final del proceso [3].

Desde el punto de vista legal, la formación integral se encuentra consagrada como un principio de la educación universitaria en Colombia, en el artículo $1 .^{\circ}$ de la Ley 30 de 1992, el cual establece que 'la educación superior es un proceso permanente que posibilita el desarrollo de las potencialidades del ser humano de una manera integral... y tiene por objeto el pleno desarrollo de los alumnos y su formación académica o profesional', y está categorizada, en el artículo $6 .^{\circ}$ de la misma Ley, como uno de los objetivos de la educación superior $y$ de las instituciones educativas que tienen la misión de prestar dicho servicio [4,5].

Por razones de coherencia interna y compatibilidad con la visión del autor acerca de la formación universitaria, en este artículo se ha decidido asumir el enfoque de formación integral propuesto por Rafael Campo y Mariluz Rodríguez, para ser utilizado como marco de referencia y como base para la formulación de una propuesta pedagógica que permita la materialización del concepto en la práctica educativa [6].

De acuerdo con Campo y Rodríguez, la formación es un elemento constitutivo de la condición humana que tiene lugar en cada persona a partir de 'un proceso mediante el cual se adopta una determinada forma, y ese proceso es el propio de la vida. Nos educamos al existir'. Sin embargo, para completar el proceso de formación desde las vivencias personales 'se necesitaría que cada ser recorriera en su vida el acumulado de humanidad y esto sería imposible; por ello, las sociedades han delegado en la institución edu- cativa la tarea de contribuir al proceso de formación de quienes van llegando al mundo' [6].

Por otra parte, calificar la formación como integral implica orientar el sentido del proceso hacia el desarrollo pleno de las potencialidades del ser humano. Una educación encaminada a la formación integral asume al individuo 'como una persona íntegra, como totalidad, es una educación que reconoce la existencia de múltiples dimensiones humanas en constante interrelación, no por partes, sino en permanente despliegue, presentes siempre en todos los ámbitos de la existencia; es una educación que reconoce que en cualquier actividad educativa está implicada la persona como un todo' [6].

En esa misma línea, Delors, en el informe a la UNESCO (1996), plantea que la educación tiene 'la misión de permitir a todos, sin excepción, hacer fructificar los talentos y capacidades de creación, lo que implica que cada uno pueda responsabilizarse de sí mismo y realizar su proyecto personal', en otras palabras, el compromiso de optimizar las capacidades del individuo para garantizar la construcción exitosa de un proyecto de vida personal y de un proyecto de sociedad que permita responder en forma adecuada a las necesidades y expectativas del colectivo al que pertenece [7].

El informe define cuatro pilares básicos de la educación, que pueden considerarse a la vez como pilares para el desarrollo de la formación integral, desde varias dimensiones: el conocimiento (aprender a conocer), la praxis (aprender a hacer), la ética (aprender a ser) y la relación con otros (aprender a convivir), elementos que coinciden plenamente con los conceptos planteados por otros autores como sustento filosófico y marco operacional para la materialización del constructo 'formación integral' en la educación superior [7].

A partir de los referentes mencionados, la formación integral no puede estar fundamentada únicamente en el desarrollo de competencias ni en la transmisión de información, sino que tiene que estar orientada hacia la adquisición de habilidades que permitan asumir la vida como una oportunidad de aprendizaje continuo, lo que determina la responsabilidad de 'propiciar el desarrollo del talento humano y permitir que cada quien sea lo que puede ser, que avive y busque perfeccionar sus fortalezas en el contexto de la totalidad de su ser' $[6,7]$. 
Coincide con lo expresado hasta aquí el planteamiento de Elsa Acevedo sobre una reflexión del filósofo Luis Enrique Orozco, cuando afirma que la universidad debe ser 'un espacio que posibilita la construcción del individuo más que la producción de profesionales', y que el desarrollo integral de los estudiantes universitarios debe estar enfocado hacia la 'formación de recursos humanos aptos para el manejo de la ciencia, la tecnología y, en general, los saberes, con criterios éticos, morales y humanistas', en otras palabras, hacia la formación de hombres y mujeres de bien antes que de excelentes profesionales en un área determinada [8].

\section{El profesionalismo médico}

El concepto de profesionalismo, entendido como el conjunto de conocimientos, habilidades, principios y valores que sustentan una práctica idónea de la medicina en el marco de los más elevados estándares de calidad científica, ética y humanística, también ha adquirido una gran relevancia en el panorama de la educación médica en los últimos años, como consecuencia de los cambios surgidos en el contexto en que se desarrollan las actividades propias del ejercicio de la medicina [2].

La comercialización de la salud, las condiciones en que se lleva a cabo la práctica profesional, la presión de gremios y asociaciones en busca de beneficios personales e institucionales, la competitividad exagerada del sector y la primacía de los intereses individuales de algunos médicos sobre los de la sociedad que demanda sus servicios se han convertido en una gran amenaza para el cometido fundamental de la medicina [9-13].

Para Swick, el profesionalismo médico debe fundamentarse en las características del área en que se desarrolla el ejercicio profesional y estrechamente relacionado con las condiciones del hacer cotidiano, desde la perspectiva individual y colectiva [14]. En coincidencia con lo anterior, Rodríguez-Portales expresa que 'la definición de profesionalismo va más allá del diccionario, que sólo indica que se trata de la competencia o destreza esperada en un individuo que ejerce una profesión. En medicina, el concepto debe afincarse en la naturaleza misma de la profesión médica, entendida ésta como una forma de vida, asentada en la ética' [15].

Pardell reafirma lo anterior y resalta la importancia de concebir una 'nueva profesión para una nueva sociedad', hecho que determina la concreción del concepto de profesionalismo médico en torno al 'conjunto de valores, actitudes y comportamientos orientados al servicio del paciente y de la sociedad antes que en beneficio propio' [12]. El código no escrito de conducta médica que llamamos profesionalismo surge a partir de la naturaleza de la medicina y sus valores permanentes, por un lado, y de la consideración de la rectitud o corrección del actuar médico, por el otro [15].

En esa misma línea, siguiendo con Rodríguez-Portales, 'la medicina tiene valores que la han caracterizado tradicionalmente, y aunque en muchas épocas estos valores han sido cuestionados y aun atacados, siempre han sobrevivido a las modas y a los cambios, principalmente por ser una forma de vida asentada en la ética. Estos valores se insertan en una antropología y una cosmovisión que, aunque puedan diferir según los individuos y las épocas, se basan en una relación de ayuda', una relación entre alguien necesitado y un 'dispensador de ayuda' en la que deben hacerse patentes 'los valores tradicionales y permanentes de la medicina, la naturaleza misma de la profesión [15].

Una concepción moderna del profesionalismo médico fue publicada por un grupo internacional de organizaciones médicas bajo el título Medical professionalism in the new millennium: a physician charter. El preámbulo del documento hace énfasis en el hecho de que el profesionalismo es la base del contrato de la medicina con la sociedad, sentencia que demanda la consideración de las necesidades del paciente sobre los intereses del médico, el mantenimiento de los máximos estándares de competencia e integridad, y la provisión de asistencia calificada a la sociedad en materia de salud [16].

En los siguientes párrafos, el documento plantea la existencia de tres principios fundamentales que sirven como sustento del profesionalismo, y establece un conjunto de responsabilidades que caracterizan la práctica médica en el contexto descrito. En cuanto a los principios, se hace mención de la primacía del bienestar del paciente sobre cualquier otro interés del médico o de las instituciones del sistema de salud; de la autonomía del paciente en la toma de decisiones relacionadas con su estado de salud y de la obligación del médico de garantizar la aplicación de los criterios de justicia social, incluyendo la distribución equitativa de los recursos disponibles para tal fin y la 
eliminación de cualquier forma de discriminación en el cuidado de la salud [16].

Por otra parte, entre las responsabilidades profesionales se encuentran el compromiso con la competencia profesional, la honestidad con el paciente, el respeto por la confidencialidad, el mantenimiento de una relación adecuada con el paciente, el mejoramiento continuo de la calidad de la atención, la garantía de un acceso equitativo a los servicios sanitarios, la distribución adecuada de los recursos disponibles en el sistema, la integridad y el uso apropiado del conocimiento científico y la tecnología, la resolución de los conflictos de interés y la autorregulación individual y colectiva de la profesión $[13,16,17]$.

Diferentes autores resaltan la importancia de incluir las competencias que sustentan el profesionalismo médico como un núcleo de formación de los estudiantes de medicina. En tal sentido, Millán et al plantean el hecho de que, a pesar de las dificultades existentes para definir con claridad las estrategias para consolidar los valores médicos, 'aspectos ciertamente intangibles de la profesión', éstos resultan imprescindibles para el ejercicio idóneo de la medicina, al proporcionar bases sólidas para afrontar las responsabilidades propias del quehacer médico [18].

\section{Las prácticas educativas frente a la formación integral y al profesionalismo médico}

En la práctica, la universidad contribuye a la formación integral y al desarrollo del profesionalismo médico en la medida en que logre involucrar a los estudiantes en vivencias intelectuales, estéticas y morales que les permitan sentirse implicados y afectivamente comprometidos', en la construcción de signos y significados compartidos para configurar un imaginario colectivo en que se desarrollen prácticas y valores específicos a partir de la diversidad, mediante el ejercicio de la docencia, la investigación y la proyección a la sociedad [9].

Para Talbott y Mallott, el humanismo médico, la bioética y el profesionalismo médico se encuentran entre los 'tópicos más candentes' de la educación médica en la actualidad y deben abordarse durante la formación profesional en los niveles de pregrado y posgrado [19]. Entre los elementos propuestos como fundamento de la formación in- tegral y pilares del profesionalismo médico estarían incluidos el altruismo, la tolerancia frente a la diversidad, la sensibilidad social, la adherencia a los principios éticos que regulan la práctica médica y la capacidad de establecer relaciones asertivas con colegas, pacientes y familiares $[19,20]$.

En ese orden de ideas, ante el reto que implica la formación de los responsables del cuidado de la salud y la generación de los espacios requeridos para la consolidación del profesionalismo médico, es necesario reflexionar acerca de los mecanismos utilizados por la universidad para lograr la transformación de 'inexpertos aprendices' en 'maestros alquimistas', y acerca del compromiso y el grado de preparación de los maestros para asumir la tarea de adaptar los sistemas educativos a las necesidades reales del mundo moderno, sin perder de vista los principios y valores que han enmarcado la práctica médica desde sus inicios [22,23].

Así, en busca del camino que conduce a la 'piedra filosofal' capaz de transformar a los jóvenes estudiantes de medicina en ciudadanos y profesionales capaces de responder en forma adecuada frente a las demandas de la sociedad, es necesario promover el diseño de ambientes de aprendizaje que estimulen el desarrollo de las funciones cognitivas, la adquisición de habilidades y destrezas, el pensamiento crítico, la autonomía en la toma de decisiones, el liderazgo, el trabajo colaborativo, la comunicación eficaz, la sensibilidad social, la solidaridad, la ética y la tolerancia frente a la diversidad [22].

\section{Formación integral y profesionalismo médico en el aula}

Los elementos que subyacen a la formación integral y en forma subsecuente al desarrollo del profesionalismo médico han sido objeto de múltiples consideraciones a través del tiempo; sin embargo, en la mayoría de los casos han estado ligados al currículo oculto o no formal. No obstante, su presencia o ausencia implícita en las acciones pedagógicas y en los actores del proceso educativo ejerce una influencia determinante en el resultado final, dada su condición de elementos paradigmáticos en el 'modelado' del estudiante en torno a los ideales de la profesión médica $[24,25]$.

La tarea de diseñar espacios educativos orientados a la formación integral y al desarrollo del 
profesionalismo médico no debería revestir dificultades significativas en la práctica; sin embargo, la complejidad de los elementos que subyacen a la consolidación de dichos conceptos y la gran variabilidad de las actividades que sustentan la formación del individuo hacen necesario emplear una metodología que permita integrar la construcción del conocimiento, la cualificación de las habilidades y destrezas propias de la práctica médica, la consolidación de los valores que sustentan el profesionalismo médico y la búsqueda de soluciones a los problemas sociales.

La propuesta para materializar en el aula los conceptos 'formación integral' y 'profesionalismo médico' se fundamenta en la utilización de la planeación estratégica como una herramienta de diseño de ambientes de aprendizaje orientados a la consolidación de las competencias en los dominios del saber, del saber hacer, del saber ser y del saber convivir, buscando garantizar que los resultados obtenidos al final del proceso educativo estén en concordancia con los objetivos planteados al inicio de las actividades académicas propuestas en el proyecto curricular que las enmarca [22].

La elaboración del plan estratégico debe incluir la formulación de objetivos permanentes (definidos a partir del proyecto educativo y de los referentes universales del ejercicio de la profesión médica), objetivos estratégicos (establecidos como metas a corto, mediano y largo plazo) y objetivos operativos (materializados en acciones específicas tendientes al cumplimiento de los dos anteriores). El procedimiento para la definición del plan estratégico incluye el análisis de los siguientes puntos $[21,26,27]$ :

- Marco de referencia: políticas institucionales, lineamientos de la facultad, propuesta curricular, referentes del desempeño profesional.

- Justificación: argumentos que sustentan la importancia de las actividades que se deben desarrollar y definición de la relación existente con el marco de referencia propuesto.

- Objetivos: intencionalidad del acto educativo y de las acciones pertinentes para su concreción.

- Contenidos temáticos: selección de unidades significativas de conocimiento de acuerdo con el área objeto de trabajo.

- Estrategias didácticas: selección de actividades tendientes a promover la adquisición de conocimientos, habilidades, principios y valores individuales y colectivos.
- Recursos de apoyo: elementos bibliográficos, audiovisuales, materiales y equipos de soporte a la actividad académica.

- Estrategias de evaluación: selección de actividades tendientes a valorar el desarrollo del individuo, el nivel de logro alcanzado y los productos concretos de la acción educativa.

- Cronograma de actividades: herramienta de planeación temporal de las actividades propuestas.

- Productos: resultados esperados como fruto del trabajo del estudiante al final del proceso.

- Aspectos afectivos y cognitivos del estudiante: condicionantes emocionales, perceptuales y cognitivos del aprendizaje.

A modo de ejemplo, se presenta a continuación el plan estratégico y la 'disección pedagógica' del espacio académico 'Teorías del envejecimiento', el cual forma parte del módulo de gerontología, en el curso de salud familiar de una reconocida facultad de medicina de la ciudad de Bogotá.

\section{'Teorías del envejecimiento': plan estratégico}

\section{Marco de referencia}

La sesión de trabajo 'Teorías del envejecimiento' forma parte integral del módulo de atención primaria en salud orientada a la población geriátrica, el cual pertenece al área de salud familiar.

Los elementos articuladores del proceso de diseño incluyen:

- Conocimiento.

- Procesos cognitivos.

- Trabajo en equipo.

- Liderazgo.

- Capacidad de resolución de problemas.

- Creatividad.

- Responsabilidad.

- Cumplimiento.

\section{Justificación}

A partir de la actividad, se busca generar una mayor comprensión de los fenómenos biológicos que influyen en el proceso de envejecimiento e introducir elementos de reflexión en torno a la importancia del estilo de vida en la calidad de la vejez. 
Por otra parte, las actividades propuestas tienen la intencionalidad de promover el desarrollo de algunas habilidades cognitivas, actitudinales, axiológicas y praxiológicas básicas para la formación integral y la consolidación del profesionalismo médico.

\section{Objetivos}

- Mejorar la comprensión de los fenómenos que determinan el proceso de envejecimiento.

- Generar una reflexión acerca de la importancia del autocuidado a lo largo del ciclo vital como estrategia de promoción de la salud.

- Promover el desarrollo de algunas habilidades que sustentan la formación integral del individuo y el profesionalismo médico.

\section{Contenidos temáticos}

- Muerte programada.

- Acumulación de errores.

- Biología y envejecimiento.

\section{Estrategias didácticas}

- Activación de motivadores intrínsecos.

- Activación de conocimientos previos.

- Aprendizaje colaborativo.

- Aprendizaje vivencial.

- Juego de roles.

- Metacognición.

\section{Recursos de apoyo}

- Material bibliográfico.

- Equipos audiovisuales.

- Papelería.

\section{Estrategias de evaluación}

- Exposición oral.

- Evaluación escrita.

- Relatoría.

- Autoevaluación individual y grupal.

- Coevaluación.

- Evaluación global de la actividad.

\section{Selección de actividades}

Las actividades seleccionadas se definen con base en los objetivos globales, estratégicos y operacio- nales, siendo incluidas de forma intencionada en el mapa de la sesión. Entre las actividades llevadas a cabo en este espacio académico se encuentran dinámicas orientadas a:

- Motivación.

- Desarrollo de procesos cognitivos.

- Desarrollo de habilidades, principios y valores personales.

- Desarrollo de habilidades de comunicación y trabajo colaborativo.

- Desarrollo de creatividad.

- Desarrollo del pensamiento crítico y la autorreflexión.

\section{Cronograma de actividades}

- Sesión 1: introducción, instrucción (tiempo estimado: $2 \mathrm{~h}$ ).

- Sesión 2: presentación, evaluación (tiempo estimado: $2 \mathrm{~h}$ ).

- Tutoría: aclaraciones, ajustes (tiempo estimado: $2 \mathrm{~h}$ ).

- Trabajo independiente: preparación (tiempo estimado: $6 \mathrm{~h}$ ).

\section{Productos}

- Material expositivo.

- Relatoría del proceso.

- Documento para publicación.

- Blog.

\section{Aspectos afectivos y cognitivos del estudiante}

Activación de motivación intrínseca, activación de conocimientos previos, aprendizaje colaborativo, adquisición de valores y habilidades, liderazgo, autoaprendizaje, manejo de recursos didácticos.

En el anexo 'Anatomía de una sesión de formación integral en medicina', se puede observar en detalle la aplicación práctica del proceso de planeación pedagógica del espacio académico referido. En la columna derecha se describen las actividades propuestas para el logro de los objetivos establecidos en el plan estratégico, y en la izquierda, la intencionalidad con que han sido introducidas en el proceso educativo y la utilidad de cada una de éstas para la formación integral del estudiante y para el desarrollo de las competencias que fundamentan el profesionalismo médico. 


\section{Bibliografía}

1. Quintana O. Los objetivos de la medicina. Revista de Calidad Asistencial 2003; 18: 132-5.

2. Wojtczak A. Profesionalismo médico: una problemática global. Educ Med 2006; 9: 144-5.

3. Tovar MC. El significado de la formación integral en estudiantes de último semestre de la Facultad de Salud de la Universidad del Valle, 2002. Colombia Med 2002; 33 : 149-55.

4. Ministerio de Educación Nacional. Ley 30 de 1992. Bogotá: Ministerio de Educación Nacional; 1992.

5. Angulo B, González H, Santamaría C, Sarmiento P. Formación integral de los estudiantes: percepción de los profesores de la Facultad de Salud de la Universidad del Valle. Colombia Med 2007; 38 (Supl 2): S15-26.

6. Campo R, Rodríguez ML. La formación integral como posibilitadora de lo humano. Formas en Educación 1999; 1: 1-32.

7. Delors J. La educación encierra un tesoro. Informe a la UNESCO de la Comisión Internacional sobre la Educación para el siglo XXI. Madrid: Santillana/UNESCO; 1996.

8. Acevedo E. La formación humana integral: una aproximación entre las humanidades y la ciencia. Organización de Estados Iberoamericanos para la Educación, la Ciencia y la Cultura. URL: http://www.oei.es/salactsi/ elsa1.htm.

9. Orozco LE. La formación integral: mito y realidad. URL: http://www.unibague.edu.co/portal/phpnews/files/ la\%20formacion20integral(1).pdf.

10. Cohen J. Teoría de las profesiones: la medicina como paradigma de profesión preeminente. URL: http://www. fundacionmhm.org/pdf/Mono7/Articulos/articulo1. pdf.

11. Murúa P. Cómo y dónde se encuentra la profesión médica. Visión de un médico especialista en formación. Educ Med 2006; 9 (Supl 1): S5-8.

12. Pardell H. El nuevo profesionalismo médico: una ideología expresada en conductas. URL: http://www.fundacionmhm.org/pdf/Mono7/Articulos/articulo2.pdf.
13. Stephenson T. Medical professionalism in the 21st century. Clin Manag 2005; 13: 1-5.

14. Swick H. Toward a normative definition of medical professionalism. Acad Med 2000; 75: 612-6.

15. Rodríguez-Portales J.A. Profesionalismo y educación médica. ARS Médica. Universidad Católica de Chile. URL: http//escuela.med.puc.cl/pub/arsmedica/ArsMedica15/ Profesionalismo.html.

16. Sox H. Medical professionalism in the new millennium: a physician charter. Ann Intern Med. 2002; 136: 243-6.

17. Whitcomb M. What does it mean to be a physician? Acad Med 2007; 82: 917-8.

18. Millán Núñez-Cortés J, García J, Calvo E, Díez R, Villanueva JL, Nogales A. La transmisión de los valores médicos: competencias específicas. Educ Med 2006; 9 (Supl 1): S24-8.

19. Talbott J, Mallott D. Professionalism, medical humanism and clinical bioethics: the new wave. Does psychiatry have a role? J Psychiatr Pract 2006; 12: 384-90.

20. Villanueva JL. Necesidad de adquirir competencias en valores y actitudes. Educ Med 2006; 9 (Supl 1): S39-41.

21. Hilton S. Medical professionalism: how can we encourage it in our students? Clin Teach 2004; 1: 69-74.

22. Morales-Ruiz J.C. Planeación estratégica orientada al diseño de ambientes de aprendizaje en la educación superior. URL: http://www.areadelaeducacion.com/planeacionestrategica.htm.

23. Monereo C, Pozo J. ¿En qué siglo vive la escuela? El reto de la nueva cultura educativa. Cuadernos de Pedagogía 2001; 298: 50-5.

24. Rosselot J. Dimensiones del profesionalismo médico: proyecciones para el siglo xxi. Rev Med Chile 2006; 134: 657-64.

25. Bernadá M. Comentario sobre 'The developing physician -becoming a professional'. Rev Med Urug 2007; 23 : 269-70.

26. López C. La misión: brújula estratégica. Gestiópolis. URL: http://www.gestiopolis.com/canales/gerencial/articulos/ 28/mision.htm.

27. López C. Análisis DOFA: una herramienta de gestión empresarial. Gestiópolis. URL: http://www.gestiopolis. com/demarketing/articulos/no\%29/dofa.htm. 


\section{Anexo. Anatomía de una sesión de formación integral en medicina.}

El concepto de formación integral en el proceso educativo asume al ser humano como una persona íntegra en la que se da una interrelación permanente de las diferentes dimensiones del ser humano; en tal sentido, la estructuración de un currículo fundamentado en formación integral busca propiciar el desarrollo armónico de todas las dimensiones del individuo con miras a la adquisición de competencia profesional, autonomía, trascendencia y justicia social.

Tema

Teorías del envejecimiento.

\section{Extensión}

Trabajo presencial: cuatro horas.

Trabajo independiente: seis horas.

Espacio de tutoría (presencial, chat académico, foros electrónicos): dos horas.

\section{Dinámica}

\section{Introducción}

Se lleva a cabo la lectura del documento 'La fuente de la eterna juventud', preparado por el docente con el objetivo de contextualizar el tema, definir los conceptos clave y preparar al estudiante para el manejo adecuado del tema.

\section{Motivación}

Lectura colectiva del texto 'La muerte de Carlos II'.

\section{Uso de activadores del conocimiento}

Definición de conceptos claves y exploración de presaberes, análisis de expectativas, establecimiento de metodología, tiempo de trabajo, negociación de reglas de trabajo.

\section{Organización de grupos de trabajo}

Distribución de los estudiantes en dos grupos de trabajo, a cada uno de los cuales se le asigna una de las dos teorías del envejecimiento:
Justificación desde el modelo educativo del programa

Establecimiento del marco de trabajo

Definición de referentes de formación

Temática propuesta en el proyecto pedagógico de aula

Definida de acuerdo con experiencias previas

Aplicación de principios pedagógicos

Uso de la tecnología informática

Motivación previa Generación de inquietudes y preguntas (incluyendo implicaciones económicas, éticas, sociales, sanitarias, entre otras)

Promoción de la motivación intrínseca mediante la narrativa

Activación de conocimientos previos

Generación de expectativas

Definición de objetivos y reglas del trabajo académico propuesto

Generación de actividades orientadas a promover

la formación integra y el profesionalismo: 
Grupo 1. Teoría de la muerte programada.

Grupo 2. Teoría de la acumulación de errores.

Nombramiento democrático de dos líderes por equipo:

Líder 1. Coordinador del equipo.

Líder 2. Observador y analista del proceso.

Líder 3. Relator

Miembros del grupo: participación responsable y equitativa, difusión de conceptos.

Entrega de guía de aprendizaje para lectura, análisis y comprensión del trabajo y de los contenidos a desarrollar durante la sesión.

Instrucciones acerca del uso de recursos didácticos de apoyo y entrega de materiales básicos.

Programación del trabajo, revisión del material, investigación autónoma, elaboración de mapa conceptual, identificación de elementos clave, reconstrucción de contenidos

Preparación de la presentación:

Tiempo (establecido previamente).

Características de la presentación.

Estándares de calidad.

Selección de expositores (uno por vía democrática, uno al azar).

Presentación de la exposición de acuerdo con las condiciones establecidas previamente.

Plenaria: puesta en común de inquietudes, preguntas del auditorio y del facilitador, discusión grupal.
Trabajo en equipo Liderazgo Seguimiento de instrucciones Manejo de grupos Capacidad de solución de problemas Creatividad Participación Responsabilidad Asignación de tareas

Habilidad lectora Aprendizaje autónomo Procesos de pensamiento

Administración de recursos Planificación

Desarrollo de la creatividad

Planeación

Habilidad lectora

Aprendizaje autónomo

Análisis, síntesis, abstracción, deducción, reconstrucción

Manejo del tiempo Administración de recursos Cumplimiento Puntualidad

Responsabilidad Organización del trabajo

Habilidades y valores profesionales

Evaluación y refuerzo de los contenidos y de las habilidades comunicativas. Comportamiento del grupo (respeto, atención, etc.)

Socialización

Aclaración de dudas Desarrollo del pensamiento inferencial 
Evaluación cuantitativa:

Prueba escrita para valoración cuantitativa de:

Conceptos claves que fundamentan las teorías.

Aplicación en otros contextos.

Presentación de informe del líder observador en relación con el proceso llevado a cabo y con los resultados obtenidos.

Evaluación cualitativa del trabajo del docente, los líderes, los miembros de cada grupo, los contenidos, la dinámica y el proceso.

Cierre de la sesión: resumen, conclusiones, refuerzo de elementos clave de aprendizaje.

Trabajo posterior:

Revisión de literatura reciente en relación con el tema mediante la ubicación y lectura de artículos científicos en otro idioma en temas definidos con el grupo: club de revistas, foro electrónico de discusión.

Elaboración de un producto final del proceso: ensayo, mapa conceptual, cuadro resumen, relatoría en un foro electrónico.

Preparación de un documento para la publicación en un medio electrónico y/o físico.
Evaluación del aprendizaje y su utilización práctica

Establecimiento del porcentaje de aprovechamiento Análisis metacognitivo del proceso y los resultados

Refuerzo del aprendizaje Metacognición de metodología de trabajo y resultados

Promoción de la investigación y la autogestión del conocimiento Uso de una segunda lengua

Uso de la tecnología informática

Sistematización de la producción grupal

Difusión a la comunidad científica

Reconocimiento del trabajo grupal 